Supporting Information

\title{
Perovskite $\mathrm{CH}_{3} \mathrm{NH}_{3} \mathrm{PbI}_{3}(\mathrm{Cl})$ single crystals: rapid solution growth, unparalleled crystalline quality and low trap density towards $10^{8} \mathrm{~cm}^{-3}$
}

Zhipeng Lian, Qingfeng Yan, * Taotao Gao, Jie Ding, Qianrui Lv, Chuangang Ning, Qiang Li, and Jia-lin Sun*

S1. Sample preparation and characterization

S2. Photographs of as-grown large MAPbI3@STL and MAPbI3@ITC single crystals

S3. Powder X-ray diffractions of as-grown crystals and Rietveld refinement for MAPbI3(Cl)

S4. X-ray diffraction for (100) facet of as-grown MAPbI3(CI) single crystal

S5. C12p core level XPS spectra for MAPbI3@STL and MAPbI3(CI)

S6. Schematic seeded growth process of large bulk crystal MAPbI3(CI)

S7. The role of chlorine on the rapid growth of $\mathrm{MAPbI}_{3}(\mathrm{Cl})$ crystals

S7.1 Representative photographs for growth process

S7.2 Solubility effect at the presence and absence of chlorine

S7.3 Thermodynamic and kinetic impact of chlorine additive on crystal growth

S7.4 Impact of chlorine on the attachments of growth species during the growth process

S8. Trap state density and carrier mobility calculations based on a standard SCLC model 


\section{S1. Sample preparation and characterization}

Raw materials: Methylamine hydroiodide was prepared by mixing stoichiometric methylamine $(40 \% \mathrm{w} / \mathrm{w}$ aqueous solution, TCI) with hydroiodic acid (57 wt.\% aqueous solution, ACROS ORGANICS) at $0{ }^{\circ} \mathrm{C}$. The product was obtained by distillation in vacuum, washing 4 times with ethyl ether (99.5\%, Sinoreagent). Hydrogen chloride $(36.0 \sim 38.0$ wt.\%), acetate trihydrate $(99.5 \%)$ and methylamine hydrochloride $(98.0 \%)$ were purchased from Sinoreagent and TCI, respectively. [6,6]-Phenyl C61 butyric acid methyl ester (99\%) was purchased from Xi'an Polymer Light Technology Corp.

Growth of $\mathrm{MAPbI}_{3}(\mathrm{Cl})$ single crystals (4 8 $\mathbf{~ m m}$ in length): $7.5 \mathrm{~g}$ lead(II) acetate trihydrate was dissolved in $30 \mathrm{ml}$ hydroiodic acid, followed by heating and stirring at $100{ }^{\circ} \mathrm{C}$ for $10 \mathrm{~min}$. A $\mathrm{MA}^{+}$mixture $(0.67 \mathrm{M})$ was prepared by dissolving methylamine hydroiodide and methylamine hydrochloride $(>98.0 \%$, TCI) in mole ratio of $1: 1 \mathrm{in} 7 \mathrm{ml}$ hydroiodic acid. The two solutions were heated to $130{ }^{\circ} \mathrm{C}$ separately, and stirred for 2 hours, then mixed together (named as growth solution). Small $\mathrm{MAPbI}_{3}(\mathrm{Cl})$ crystals were prepared via spontaneous crystallization by rapid lowering the solution temperature from $108{ }^{\circ} \mathrm{C}$ to $60^{\circ} \mathrm{C}$.

Seeded growth of large $\mathrm{MAPbI}_{3}(\mathrm{Cl})$ single crystal (size: $20 \mathrm{~mm} \times 18 \mathrm{~mm} \times 6 \mathrm{~mm}$ ): Bulk single crystals of $\mathrm{MAPbI}_{3}(\mathrm{Cl})$ were grown via the bottom-seeded solution growth method. A carefully selected seed crystal was placed in a borosilicate glass bottle (purchased from Cleman Chemical) then preheated slowly to $105^{\circ} \mathrm{C}$ in an oil-bath. Then the aforementioned growth solution was quickly poured into the bottle (a schematic of synthesis process is shown in Figure S5). The temperature was kept $105^{\circ} \mathrm{C}$ for $1 \mathrm{~h}$ to dissolve the outer surface of the seed crystal, then it quickly dropped to $100{ }^{\circ} \mathrm{C}$ and followed by a cooling rate of $0.2{ }^{\circ} \mathrm{C} / \mathrm{h}$ for $12 \mathrm{~h}$, then $0.5^{\circ} \mathrm{C} / \mathrm{h}$ to $60{ }^{\circ} \mathrm{C}$, and whereafter $1{ }^{\circ} \mathrm{C} / \mathrm{h}$ to $40{ }^{\circ} \mathrm{C}$. In this period, small crystal grew into large size. Altogether, it took about 5 days to grow the large $20 \mathrm{~mm} \times 18 \mathrm{~mm} \times 6 \mathrm{~mm}$ single crystal of $\mathrm{MAPbI}_{3}(\mathrm{Cl})$. The average growth rate was estimated around $18 \mathrm{~mm}^{3} \mathrm{~h}^{-1}$. It's worth noting that during the seed crystal growing into large, a spontaneous heterogeneous 
nucleation might occur on the bottom of glass bottle, because the presence of impurity particles, ions or rough surfaces will stimulate the nucleation. Such effects may affect the large single crystal growth. In order to minimize the side nucleation on the bottom of the bottle, the two factors should be carefully considered. Firstly, the substrate (bottom of the bottle) should be smooth as much as possible to reduce the nucleation sites. Secondly, the growth solution should be heated well (when all visible particles disappeared, continue heating the growth solution at $120 \sim 130{ }^{\circ} \mathrm{C}$ for $20 \mathrm{~min}$ ) to make sure a thorough dissolution.

Crystal growth of $\mathrm{MAPbI}_{3} @$ @SL and $\mathrm{MAPbI}_{3} @ \mathbf{I T C}_{\mathbf{T}}: \mathrm{MAPbI}_{3} @ \mathrm{STL}$ single crystals were grown by using the bottom-seeded solution growth (BSSG) method, the detailed preparation process was described in our previous work. ${ }^{1}$ A large $\mathrm{MAPbI}_{3} @ \mathrm{STL}$ single crystal is shown in Figure $\mathrm{S} 1 \mathrm{a}$, with the length exceeding $12 \mathrm{~mm} . \mathrm{MAPbI}_{3} @ \mathrm{ITC}$ single crystals were grown via the method reported by Liu. ${ }^{2} \mathrm{MAI}$ and $\mathrm{PbI}_{2}$ (1.2:1) were mixed and dissolved in gamma-butyrolactone (GBA) as the precursor solution. Small MAPbI 3 @ITC single crystals with size of $\sim 3 \mathrm{~mm}$ were prepared by keeping precursor solution at $100{ }^{\circ} \mathrm{C}$ for $24 \mathrm{~h}$. By placing a seed crystal in $20 \mathrm{~mL}$ of precursor solution and keeping it at $100{ }^{\circ} \mathrm{C}$ for $60 \mathrm{~h}$, the seed grew into a large perovskite crystal with the length exceeding $12 \mathrm{~cm}$, as shown in Figure S1b.

Crystal characterization: The powder X-ray diffractions (PXRD) were collected using an X-ray diffraction spectroscopy (D8 Advance, Brüker) operated with $\mathrm{Cu}$ Ka radiation at $40 \mathrm{kV}$ and $40 \mathrm{~mA}$. The orientation for as-grown single crystal of $\mathrm{MAPbI}_{3}(\mathrm{Cl})$ was performed by XRD (D8 Advance, Brüker). Rietveld refinement of the PXRD data were performed with the General Structure Analysis System (GSAS) software. ${ }^{3}$ High resolution X-ray rocking curves were characterized using high resolution X-ray diffractometry (D8 Discover, Brüker). Photographs of as-grown crystals were taken by a digital camera (EOS 600D, Canon). Photographs to record rapid solution growth process were obtained via a camera (Pro C920, Logitech). Optical microscopic images were taken by an optical microscope (BX51TRF, 
Olympus) that was connected to a CCD camera (Pixelink-B742) and a computer for image recording. XPS spectra were measured by an X-ray photoelectron spectrometer (PHI Quantro SXM, ULVAC-PHI) with Ar ion gun sputtering on the surface. The depth profiles of the as-grown single crystals were analyzed using time-of-flight secondary ion mass spectrometry (Model TOF-SIMS 5, ION-TOF GmbH), where the pulsed primary ions from a $\mathrm{Cs}^{+}$ liquid-metal ion gun were used.

Device fabrication and characterization. Single crystal plates were prepared via mechanical polishing, then two types of devices were fabricated through thermal evaporation method. Mask with area 5-8 $\mathrm{mm}^{2}$ was used to cover the electrode to define the device area. For dark $I-V$ measurements, six nominally identical devices were fabricated for $\operatorname{MAPbI}_{3}(\mathrm{Cl})$, MAPbI ${ }_{3} @ \mathrm{STL}$ and $\mathrm{MAPbI}_{3} @ \mathrm{ITC}$ respectively.100 nm Au electrodes were deposited on the top and bottom surfaces of as-polished $1.13 \mathrm{~mm}$-thick single crystal plates. For transient photovoltaic analysis, $174 \pm 4 \mu \mathrm{m}$-thick crystal plates were prepared, followed by a vacuum thermal evaporation of $25 \mathrm{~nm}$ Au electrodes on the top surface and then sequential depositions of $10 \mathrm{~nm}$ PC61BM as electron transfer layer and $100 \mathrm{~nm}$ Al electrode on bottom surface (vacuum : $1.2 \times 10^{-3} \mathrm{~Pa}$ ). Dark $I-V$ curves were collected by a Keithley 2400 sourcemeter. In TPV measurements, we applied a laser pulses $(532 \mathrm{~nm}, 5 \mathrm{~ns}$ width from a diode pumped Q-switched Nd: YAG laser model NL202) to pass through the semi-transparent Au electrode and then irradiated on the top surface of single crystals to generate a transient photovoltaic signal. The transient voltage signals were recorded by a PicoScope 4227 oscilloscope (1 GHz, 12Bit) with a large resistance of $1 \mathrm{M} \Omega$. A simulated open circuit condition was thus formed where the photo-generated charge carriers can be effectively blocked. 


\section{S2. Photographs of as-grown large $\mathrm{MAPbI}_{3} @ S T L$ and $\mathrm{MAPbI}_{3} @ I T C$ single crystals}

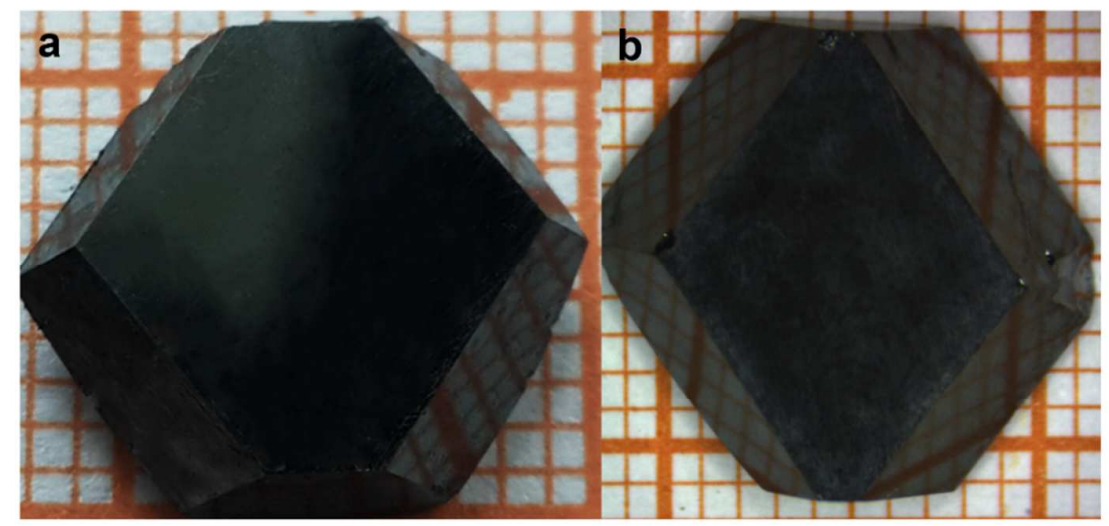

Figure S1. Photographs of $\mathrm{MAPbI}_{3} @ \mathrm{STL}$ (a) and MAPbI ${ }_{3} @ \mathrm{ITC}$ (b) bulk single crystals. The unit length of each grid on the coordinate paper is $1 \mathrm{~mm}$.

\section{S3. Powder X-ray diffractions of as-grown crystals and Rietveld refinement for $\operatorname{MAPbI}_{3}(\mathbf{C l})$}

To characterize the phase structures of the as-prepared samples, single crystals were ground thoroughly in an agate mortar, then characterized by powder X-ray diffraction (PXRD). It was observed that the peak intensity corresponding to the (110) and (220) diffractions increased gradually for as-grown $\mathrm{MAPbI}_{3} @ \mathrm{ITC}, \mathrm{MAPbI}_{3} @ \mathrm{STL}$ and $\mathrm{MAPbI}_{3}(\mathrm{Cl})$, as shown in Figure S2a, implying an increase in crystallinity. In order to confirm the crystal structure of $\mathrm{MAPbI}_{3}(\mathrm{Cl})$, Rietveld structure refinement was performed by using the powder X-ray diffraction (XRD) data. The reported $\mathrm{I} 4 / \mathrm{mcm}$ structure was introduced as an initial model in the Rietveld analysis. Figure S2b shows the Rietveld fit of XRD pattern. The final refined results of unit cell parameters and reliability factors for as-grown $\mathrm{MAPbI}_{3}(\mathrm{Cl})$ are listed in Table S1. 



Figure S2. (a) Powder X-ray patterns of as-grown MAPbI $3 @ \mathrm{ITC}_{3}, \mathrm{MAPbI}_{3} @ \mathrm{STL}$ and $\mathrm{MAPbI}_{3}(\mathrm{Cl})$, showing an gradually increasing peak intensity for (110) and (220) diffractions. (b) Experimental (crosses) and calculated (red solid line) XRD patterns and their difference (blue solid line) for the Rietveld fit of $\mathrm{MAPbI}_{3}(\mathrm{Cl})$ XRD pattern by GSAS program. The short vertical lines show the position of Bragg reflections of the calculated pattern.

Table S1. Crystallographic data for $\mathrm{MAPbI}_{3}(\mathrm{Cl})$ based on Rietveld refinement.

\begin{tabular}{|c|c|c|c|}
\hline Sample & \multicolumn{3}{|c|}{$\mathrm{MAPbI}_{3}(\mathrm{Cl})$} \\
\hline Cell parameters & $\alpha=90^{\circ}$ & $\beta=90^{\circ}$ & $\gamma=90^{\circ}$ \\
\hline Space group & & mcm & \\
\hline $\mathrm{a} / \mathrm{b}(\AA)$ & & $468(16)$ & \\
\hline$c(\AA)$ & & $530(4)$ & \\
\hline $\mathrm{V}\left(\AA^{3}\right)$ & & $12(5)$ & \\
\hline \multicolumn{4}{|c|}{ Reliability factors } \\
\hline $\mathrm{R}_{\mathrm{wp}}(\%)$ & & 9.52 & \\
\hline $\mathrm{R}_{\mathrm{p}}(\%)$ & & 3.79 & \\
\hline$\chi^{2}$ & & 1478 & \\
\hline
\end{tabular}


S4. X-ray diffraction for (100) facet of as-grown $\mathrm{MAPbI}_{3}(\mathrm{Cl})$ single crystal

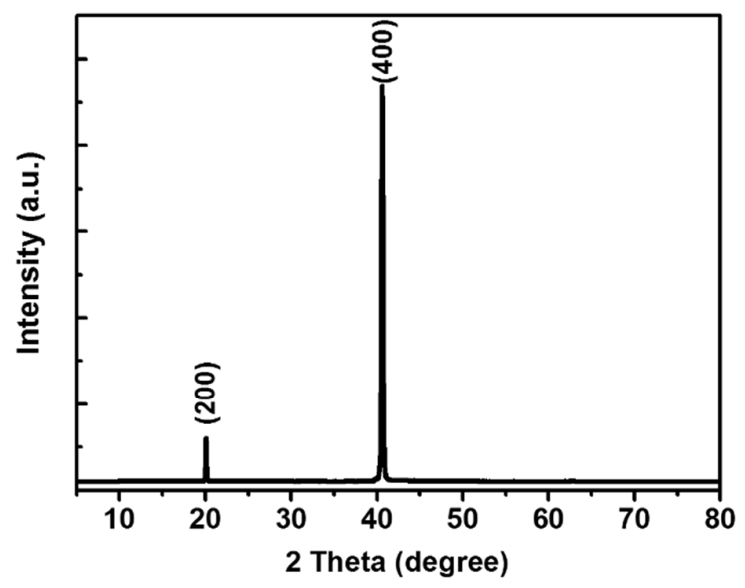

Figure S3. X-ray $2 \theta$ scan for $(100)$ facet of as-grown $\mathrm{MAPbI}_{3}(\mathrm{Cl})$ single crystal.

S5. Cl2p core level XPS spectra for $\mathrm{MAPbI}_{3} @ S T L$ and $\mathrm{MAPbI}_{3}(\mathrm{Cl})$
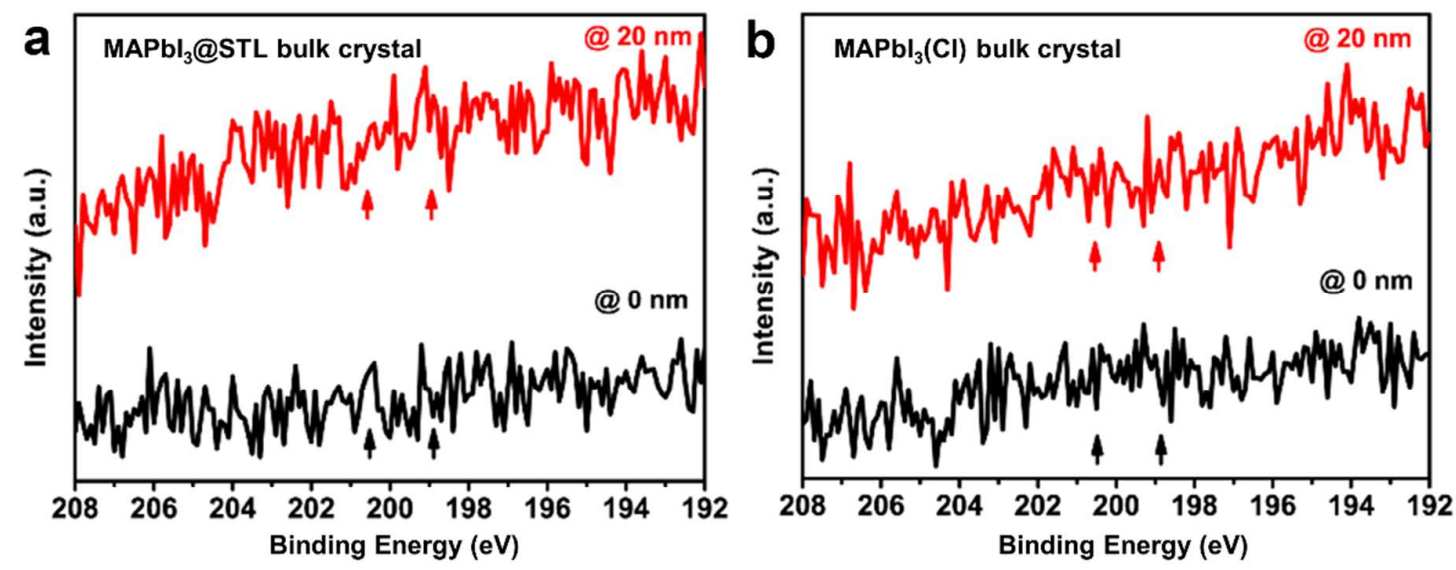

Figure S4. C12p core level XPS spectra under Ar ion gun sputtering on the perovskite single crystal $\mathrm{MAPbI}_{3} @ \mathrm{STL}(\mathrm{a})$ and $\mathrm{MAPbI}_{3}(\mathrm{Cl})$ (b). The characteristic peak binding energy of $\mathrm{Cl} 2 \mathrm{p}_{3 / 2}(198.9 \mathrm{eV})$ and $\mathrm{Cl} 2 \mathrm{p}_{1 / 2}(200.5 \mathrm{eV})$ are highlighted by the arrows. "@ 0 nm" represents the top surface of the crystal before Ar ion gun sputtering, and "@, 20 nm" refers to sputtering $20 \mathrm{~nm}$ in depth. 
S6. Schematic seeded growth process of large bulk crystal $\mathrm{MAPbI}_{3}(\mathrm{Cl})$
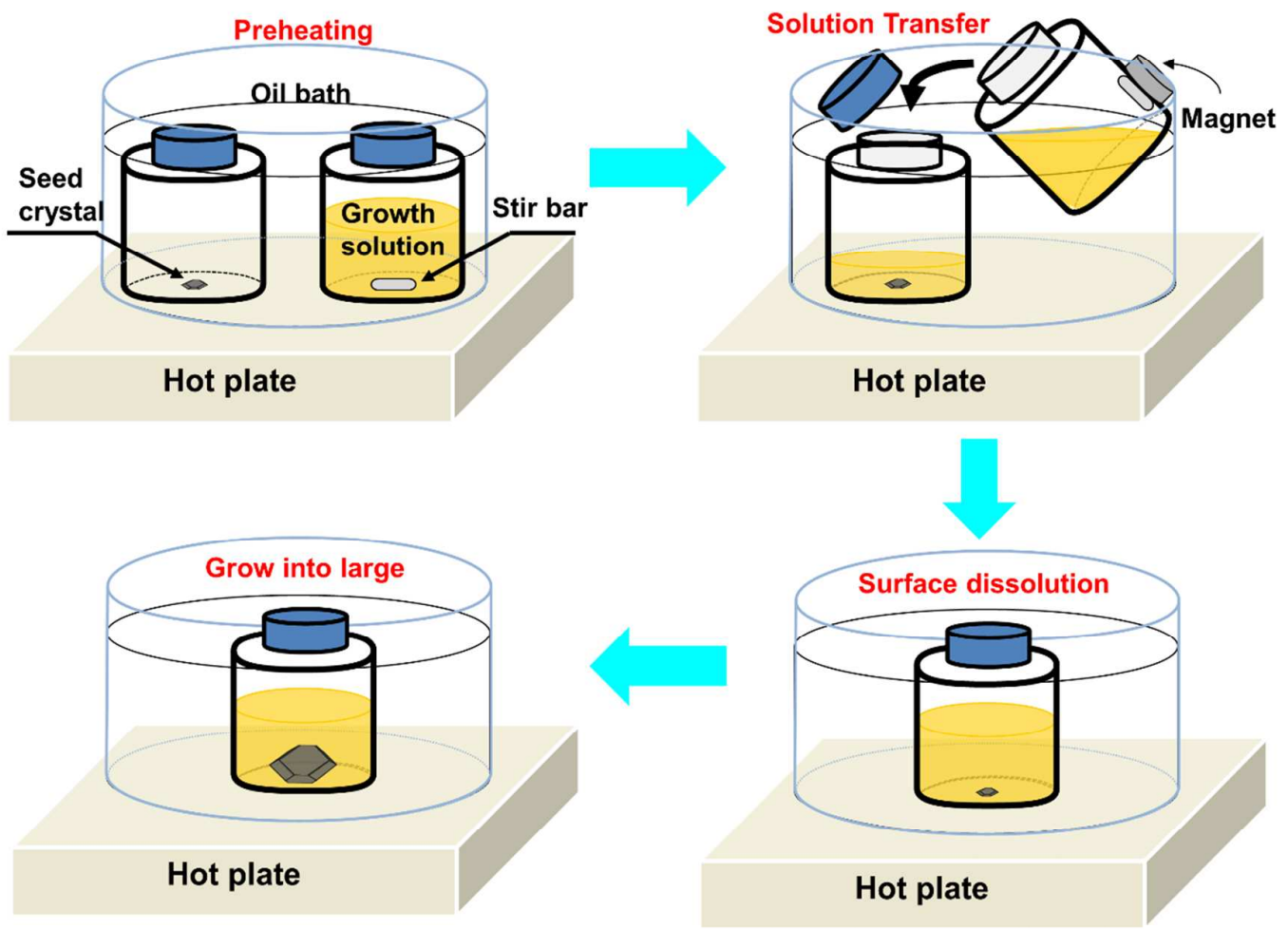

Figure S5. Schematic of seeded growth of large $\mathrm{MAPbI}_{3}(\mathrm{Cl})$ bulk single crystals. 
S7. The role of chlorine on the rapid growth of MAPbI3(CI) crystals

\section{S7.1 Representative photographs for growth process}

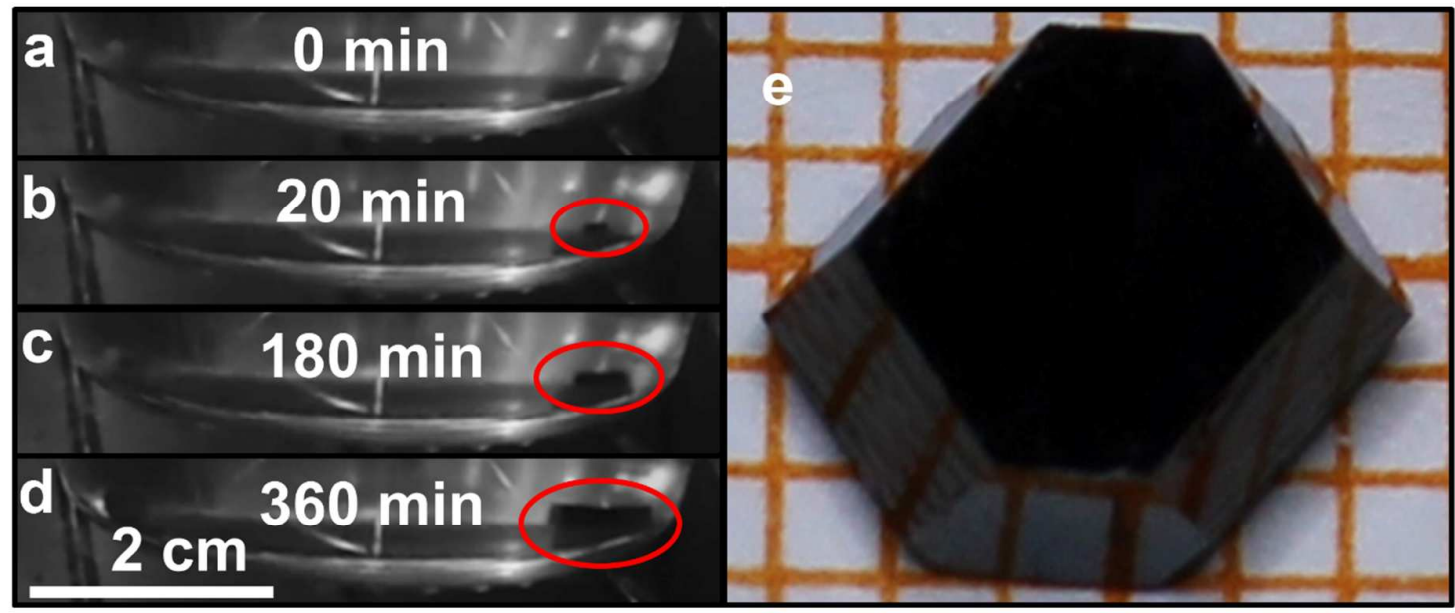

Figure S6. (a-d) Representative photos showing the rapid solution growth of $\mathrm{MAPbI}_{3}(\mathrm{Cl})$ single crystal. Within 6 hours, high-quality $\mathrm{MAPbI}_{3}(\mathrm{Cl})$ crystal was grown to a size of $\sim 5$ $\mathrm{mm}$ in length. The crystal looks larger in real observation because of the magnifying effect in solution. (e) Photograph of the as-grown dodecahedral crystal in (d).

\section{S7.2 Solubility effect at the presence and absence of chlorine}

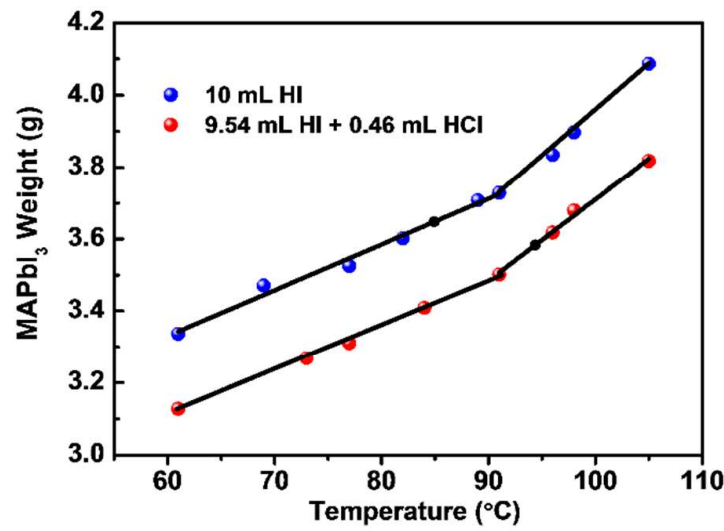

Figure S7. Solubility curves for $\mathrm{MAPbI}_{3}$ in $\mathrm{HI}$ (57 wt.\%, blue dots) and a mixture of $\mathrm{HI}$ and $\mathrm{HCl}(36.0 \sim 38.0 \mathrm{wt} . \%$, red dots) solutions. 
Solution crystal growth is a highly complex process and depends on various growth parameters such as the quality of the seed, the growth temperature, the temperature lowering rate, the character of the solution, and stirring of the solution. To understand the role of extrinsic chlorine ions in crystallization, it is necessary to measure the solubility curves at the presence and absence of chlorine ions. As shown in Figure S7, there is an obvious reduction in $\mathrm{MAPbI}_{3}$ solubility at the presence chlorine ions, which results in higher crystallization temperature because the supersaturation will be reached earlier at high temperature. The solubility in either $\mathrm{HI}(57 \mathrm{wt.} \%)$ or $\mathrm{HI}(57 \mathrm{wt} . \%)+\mathrm{HCl}(36.0 \sim 38.0$ wt.\%) solution increased linearly as the temperature increased from 60 to $90{ }^{\circ} \mathrm{C}$ and 90 to $105^{\circ} \mathrm{C}$, where $90{ }^{\circ} \mathrm{C}$ could be regarded as the turning point. Above this point, the slope of the solubility curve was markedly elevated, and the growth rate would be much rapid because the solubility variation was more sensitive and the growth species moved faster at higher temperature. However, for growth of large single crystal it was not suitable to substantially accelerate the growth rate at high temperature (above $105^{\circ} \mathrm{C}$, experimentally). In this case, the solution was unstable and tended to spontaneous nucleate. Therefore a considerable excess of chlorine largely reduced the solubility thus made the crystals outgrow. We observed the evolution of surface morphologies for $\mathrm{MAPbI}_{3}(\mathrm{Cl})$ single crystal rapidly grown from solutions with different content of $\mathrm{MACl}$ additive (Figure S8). An appropriate mole mixing ratio of $\mathrm{MACl}$ : MAI toward uniform crystal surface morphology was estimated in the range of 7:5 3:5. With a right content of chlorine additive, the arrangement of molecules in $\mathrm{MAPbI}_{3}(\mathrm{Cl})$ bulk single crystals was well-ordered. 

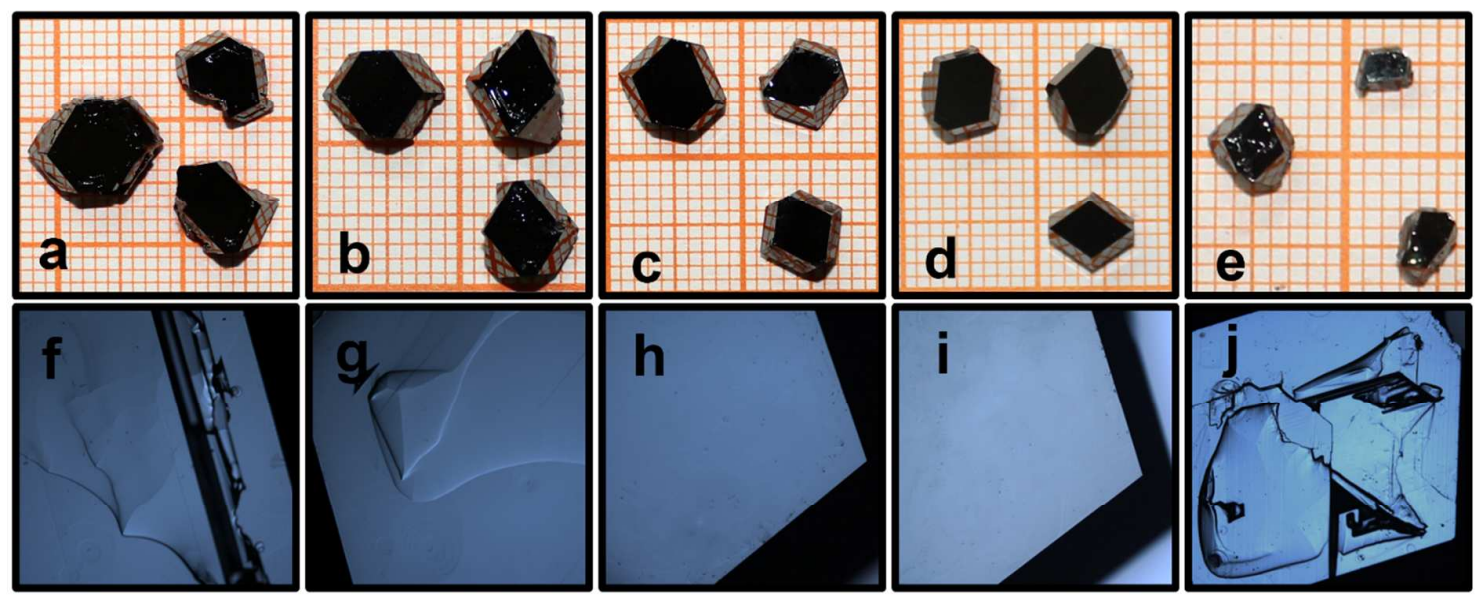

Figure S8. Photographs (a-e) and microscopic images ( $f-j)$ for surface morphologies of $\mathrm{MAPbI}_{3}(\mathrm{Cl})$ single crystals grown from solutions with different content of MACl additive. Molar ratios of MAI : MACl are 1:0 (a and f) , 19: 5 (b and g), 7:5 (c and h), 3:5 (d and i), and $1: 5$ (e and $\mathrm{j}$ ), respectively.

\section{S7.3 Thermodynamic and kinetic impact of chlorine additive on crystal growth}

Usually it is not possible to predict how a given extrinsic ions will act in a given system. From thermodynamic and kinetic perspective, the addition of chlorine might have two opposite effects during the complex crystallization process. Firstly the growth rate is affected by the kinetic constant and the step velocity. ${ }^{4}$ Chlorinated molecules adsorbed on the surface may reduce the kinetic constant and prevent the advances of the steps on the adsorption site. The retardation factor of the steps is described as: ${ }^{4}$

$$
\beta_{s t} \cong \beta_{s t}^{0}\left(1-\frac{4 d}{\left(\kappa^{*}\right)^{2}}\right)
$$

where $\beta_{s t}^{0}$ represents the retardation factor without chlorine impurities adsorption at the step, $d$ is the density of adsorbed chlorine impurities $\left(\mathrm{cm}^{-2}\right)$, and $\kappa^{*}$ is the critical curvature of the step $\left(\mathrm{cm}^{-1}\right)$. Therefore, the growth rate is reduced. However, with the addition of chlorine, the surface free energy $\gamma$ and the edge free energy $\rho$ were changed. By using a Langmuir-type isotherm and equilibrium assumption between the chlorinated molecules adsorbed on the steps and in the bulk, the resulting edge free energy is expressed by: 4,5 


$$
\rho_{\text {Chlorine }}=\rho-k_{B} T \ln C_{\text {Chlorine }}
$$

Given that the edge free energy $\rho_{\text {Chlorine }}$ decreases with $C_{\text {Chlorine }}$, the growth rate of $\mathrm{MAPbI}_{3}(\mathrm{Cl})$ crystal face will increase.

\section{S7.4 Impact of chlorine on the attachments of growth species during the growth process}

To understand the role of chlorine on growth rate, the solubility curves of $\mathrm{MAPbI}_{3}$ were measured in the presence and absence of chlorine (see S7.2). However, the solubility effect does not explain how chlorine additive contributes to the high crystalline quality in $\mathrm{MAPbI}_{3}(\mathrm{Cl})$ single crystal. In fact, with the addition of chlorine, the growth kinetics for all types of faces in the growth form of $\mathrm{MAPbI}_{3}$ crystal will be affected. According to the periodic bond chains (PBC) theory by Hertman, ${ }^{6} \mathrm{~F}$ faces of a crystal are smooth on a molecular level with pretty low kink densities, while K and S faces are rough and contain high density of kinks. Because kinks are necessary for the attachment of incoming growth species, crystal growth on $\mathrm{F}$ faces is normally hampered unless kinks are produced, which can be accomplished by either formation of dislocations from the crystal matrix or generation of some two-dimensional (2D) nuclei on the growth layer. ${ }^{6,7}$ Hence, for crystal growth on $\mathrm{F}$ faces of $\mathrm{MAPbI}_{3}$ in the absence of $\mathrm{MACl}$, mechanism of crystal growth can be regard as a dislocation growth model as schematically shows in Figure 6a-c. However, considering the interactions between crystal surface and growth species, an alternative growth process on $\mathrm{F}$ face might exist with the addition of $\mathrm{MACl}$ (Figure 6d-f).

During the growth of $\mathrm{MAPbI}_{3}(\mathrm{Cl})$ bulk crystal, the kinetic process for extrinsic chlorine might involve four stages: (I) Adsorption (temporary capture) of chlorinated molecules diffusing from the solution at the surface; (II) Migration of chlorinated molecules on the surface; (III) Adsorption (temporary or steady capture) at kink-free areas and at kinks in steps; (IV) Desorption from the surface or the steps and diffusing into the solution. The surface kinetic 
process strongly depends on the chemical nature of each component and the interaction between them. Considering the structural similarity between chlorinated molecules and iodinated molecules, it is possible that there exist a variety of reversible chlorinated intermediate molecular reactions. These reactions occur reversibly and transiently, thus making the chlorine much active on the growth surface of $\mathrm{MAPbI}_{3}(\mathrm{Cl})$ crystal. Correspondingly, the kinetic processes of adsorption, migration and desorption can occur frequently. Along with these processes, 2D nuclei induced kinks are created on the growth surface of $\mathrm{MAPbI}_{3}(\mathrm{Cl})$ crystal and provide an alternative growth process for $\mathrm{MAPbI}_{3}(\mathrm{Cl})$ single crystal, as schematically shown in Figure 6d-f. Fortunately, desorption of chlorinated molecules is rapid enough that the vast majority of chlorinated molecules can diffuse back into solution rather than enter into the crystal matrix. Therefore, crystal structure of $\mathrm{MAPbI}_{3}$ is not destroyed. Only trace amount of chlorine were steady captured, which were detected by ToF-SIMS (see Figure 5). As a result, instead of forming dislocations to realize growth species assembling, the chlorine additive provided an aided way for crystal growth on $\mathrm{F}$ faces thus reduced the dislocations in the crystal. 


\section{S8. Trap state density and carrier mobility calculations based on a standard SCLC}

\section{model}

As shown in Figure 3a, dark $I-V$ trace of $\mathrm{MAPbI}_{3}(\mathrm{Cl})$ single crystal exhibiting three different

regimes, marked for Ohmic $\left(I \propto V^{n=1}\right)$, trap-filled $\left(I \propto V^{n>3}\right)$, and Child's $\left(I \propto V^{n=2}\right)$ regime.

The critical point from Ohmic to trap-filled regime is known as trap-filled limit voltage, $\mathrm{V}_{\mathrm{TFL}}$, which is determined by the trap-state density and can be expressed as following equation: ${ }^{8}$

$$
V_{T F L}=\frac{e n_{\text {trap }} L^{2}}{2 \varepsilon \varepsilon_{0}}
$$

where $L$ is the thickness of the single crystal, $\varepsilon$ equals to 32 and represents the relative dielectric constant of $\mathrm{MAPbI}_{3}$, and $\varepsilon_{0}$ is the vacuum permittivity. When operating in Child's region, $\mathrm{I}-\mathrm{V}$ trace is conformed to Mott-Gurney's power law: ${ }^{8}$

$$
J_{D}=\frac{9 \varepsilon \varepsilon_{0} \mu V_{b}^{2}}{8 L^{3}}
$$

where $J_{d}$ represents the dark current density and $V_{b}$ is the applied voltage. We could conservatively estimate the carrier mobility in this region.

\section{References}

(1) Lian, Z.; Yan, Q.; Lv, Q.; Wang, Y.; Liu, L.; Zhang, L.; Pan, S.; Li, Q.; Wang, L.; Sun, J.-L. Sci. Rep. 2015, 5, 16563.

(2) Liu, Y.; Yang, Z.; Cui, D.; Ren, X.; Sun, J.; Liu, X.; Zhang, J.; Wei, Q.; Fan, H.; Yu, F. Adv. Mater. 2015, 27, 5176.

(3) Larson, A. C.; Von Dreele, R. B. General Structure Analysis System. LANSCE, MS-H805, Los Alamos, New Mexico 1994.

(4) Dhanaraj, G.; Byrappa, K.; Prasad, V.; Dudley, M. Springer handbook of crystal growth; Springer Science \& Business Media, 2010.

(5) Davey, R. Industrial Crystallization 1979, 78, 169.

(6) Hartman, P.; Perdok, W. Acta Crystallogr. 1955, 8, 49.

(7) Sangwal, K. J. Cryst. Growth 1999, 203, 197.

(8) Dong, Q.; Fang, Y.; Shao, Y.; Mulligan, P.; Qiu, J.; Cao, L.; Huang, J. Science 2015, $347,967$. 